\title{
TRANSPORT AND MAGNETORESISTANCE IN LOW CARRIER DENSITY FERROMAGNETS
}

\author{
P.B. LiTTLEWOOD
}

Cavendish Laboratory, Madingley Road, Cambridge CB3 0HE, England and Bell Laboratories, Lucent Technologies, Murray Hill, NJ 07974, USA

In metallic magnets, scattering from magnetic fluctuations above and near $T_{\mathrm{C}}$ may provide a substantial contribution to the electrical resistance $\rho(T)$. However, this effect is usually small because the dominant fluctuations are near $q \approx 0$, which does not produce substantial backscattering across the Fermi surface unless $2 k_{F}$ is itself small; such a situation can be realised in metallically-doped ferromagnetic semiconductors. A simple adaptation of the theory of deGennes and Friedel shows the low field magnetoresistance scales with the ratio of field induced magnetisation $m(H)$ to the saturation magnetisation $m_{\text {sat }}: \Delta \rho / \rho \approx C\left(m / m_{\text {sat }}\right)^{2}$, where $C \approx x^{-2 / 3}$, with $x$ the number of charge carriers per magnetic unit cell. Comparison to data on very different ferromagnetic metals and doped semiconductors is in broad quantitative agreement with this trend, with the prime exception of the perovskite manganese oxides, already understood to involve the extra physics of dynamic lattice distortions. At very low doping, the physics should involve ferromagnetic polarons, and polaron formation and transport are discussed.

PACS numbers: $75.70 . \mathrm{Pa}$

\section{Introduction}

"Colossal" magnetoresistance (CMR) in some manganese oxides (such as the perovskite $\mathrm{La}_{1-x} \mathrm{Sr}_{x} \mathrm{MnO}_{3}$ and the pyrochlore $\mathrm{Tl}_{2} \mathrm{Mn}_{2} \mathrm{O}_{7}$ ) has been found to accompany a transition from a metallic ferromagnetic low temperature phase to a paramagnetic high temperature phase [1]. In these materials the electrical transport is strongly influenced by the degree of magnetic order, and in addition there is a strong influence from competing charge and orbital order.

The field is very active and there are many aspects to the problem. Here we shall review just a few topics and comment on some recent developments.

The first question is how large a magnetoresistance can one expect from elementary models of magnetic scattering [2-4]. In the simplest situation, carriers are strongly scattered by magnetic fluctuations that are enhanced close to the magnetic Curie temperature $T_{\mathrm{C}}$. The suppression of the magnetic fluctuations in a small magnetic field leads to a large negative magnetoresistance above $T_{\mathrm{C}}$. This 
magnetic scattering mechanism is not different in principle from that in more familiar metallic ferromagnets such as $\mathrm{Ni}$ and $\mathrm{Fe}$, and other perovskite ferromagnets (e.g. $\mathrm{La}_{1-x} \mathrm{Ca}_{x} \mathrm{CoO}_{3}$ ), or the doped magnetic semiconductors $\mathrm{Ge}_{1-x} \mathrm{Mn}_{x} \mathrm{Te}$ and $\mathrm{Eu}_{1-x} \mathrm{Gd}_{x} \mathrm{Te}$, yet the size of the effect differs by at least an order of magnitude. It turns out that simple Born scattering can give a very good account of the magnetoresistance of many ferromagnetic metals (as already known), but especially down to low densities when the effects can be very large.

The second aspect refers to the low carrier density limit in these magnetic models, when the carrier should bind to spin distortions to produce a magnetic polaron. This is a familiar problem for an antiferromagnetic spin background, but the case of $\mathrm{Tl}_{2} \mathrm{Mn}_{2} \mathrm{O}_{7}$ may provide a rare example of the ferromagnetic case.

The third topic to be touched on is the role of charge and orbital order in the perovskite manganites. Here purely magnetic mechanisms are not appropriate, because of the strong coupling to local lattice distortions via the orbital degrees of freedom. At the most elementary level, this can simply be regarded as a competition between the delocalising effects of the electron kinetic energy, and the tendency to localise into nominally $\mathrm{Mn}^{3+}$ and $\mathrm{Mn}^{4+}$ ions that is strongly abetted by the local lattice (Jahn-Teller) distortions of the oxygen octahedra.

\section{Magnetic scattering in the Born approximation}

Scattering from magnetic fluctuations is a component of the electrical resistance. Since the fluctuations at a ferromagnetic transition lie near $q \sim 0$, they have usually only a weak effect on transport because it is primarily modes that can scatter backwards across the Fermi surface that are important, i.e. with momenta of order $2 k_{\mathrm{F}}\left(k_{\mathrm{F}}=\left(3 \pi^{2} n\right)^{1 / 3}\right.$ is the Fermi momentum in an electron gas of density $n$ ). The obvious and interesting exception is a low density electron system $k_{\mathrm{F}} a \ll 1$ (where $a$ is the lattice constant), and it is in this low density regime that the effects are largest. A fuller discussion is given in Refs. [2,3] and here just the main results are quoted.

Consider a model of a single band of carriers with hopping $t$ coupled to a ferromagnetic spin model with an exchange constant $J$

$$
H=\sum_{k} \epsilon_{k} c_{k}^{\dagger} c_{k}-J \sum_{i} \sigma_{i} S_{i}-J_{\mathrm{F}} \sum_{i j} S_{i} S_{j}
$$

Here $\sigma_{i}=c_{i, \alpha}^{\dagger} \sigma_{\alpha, \beta} c_{i, \beta}$ is the spin of the itinerant electron, and $S_{i}$ refers to the localised core spin.

A simple scaling form for the magnetic fluctuations generates an effective static potential that scatters the carriers. Thus we regard $J_{F}$ as having independent origin, not mediated by the carriers. This is strictly true in some materials $\left(\mathrm{Tl}_{2} \mathrm{Mn}_{2} \mathrm{O}_{7}, \mathrm{Cd}_{2} \mathrm{Cr}_{2} \mathrm{Se}_{4}\right)$ while inappropriate in others (double-exchange and RKKY magnets). But decoupling the origin of the magnetism from its effects on transport is reasonable.

The scattering rate, normalised to its high temperature value (corresponding to uncorrelated spins), is in the Born approximation given by $[5,6]$

$$
\tau^{-1} / \tau_{0}^{-1} \sim \int_{0}^{\pi} \chi\left(q=2 k_{\mathrm{F}} \sin (\theta / 2)\right)(1-\cos \theta) \sin \theta \mathrm{d} \theta
$$


where $q$ is the momentum transfer for this on-shell scattering, and $\chi(q)$ is the spin-spin correlation function. The integral is dominated by large scattering angles, and to study trends it is sufficient to replace $\theta$ by $\pi$ in Eq. (1). Then the magnetoresistance is

$$
\Delta \rho / \rho \approx 1-\chi\left(2 k_{\mathrm{F}}, T, H\right) / \chi\left(2 k_{\mathrm{F}}, T, 0\right)
$$

at a temperature $T$ and magnetic field $H^{*}$.

We make a simple Ginzburg-Landau approximation for the low-field magnetic susceptibility in the paramagnetic state, viz. $\chi^{-1}(q, T, H) \propto A(T)+$ $\left(q \xi_{0}\right)^{2}+\left[m(H) / m_{\text {sat }}\right]^{2}$, where $A(T)$ vanishes at $T_{\mathrm{C}}$. The correlation length $\xi(T)=$ $\xi_{0} / A(T)^{1 / 2}$ diverges as $T \rightarrow T_{\mathrm{C}}$; the corresponding divergence in $\chi$ is cutoff either by the momentum $q$, or by the field-induced magnetisation $m(H)$. The scale $m_{\text {sat }}$ is comparable to the saturation magnetisation in a large field. Using Eq. (3), we get at low fields

$$
\Delta \rho / \rho=C\left(m / m_{\text {sat }}\right)^{2} \approx\left(1 / 2 k_{\mathbf{F}} \xi_{0}\right)^{2}\left(m / m_{\text {sat }}\right)^{2}
$$

provided $k_{\mathrm{F}} \xi(T) \gg 1$. This formula depends solely on elementary parameters of the magnetic system, the bare correlation length (of order the separation between magnetic moments) and the saturation magnetisation. The use of the Ginzburg-Landau approximation for the correlation function will lead to a cusp in the resistance at $T_{\mathrm{C}}$, which is unphysical. A better theory [5] for $\chi$ leads to a peak in $\tau^{-1}$ above $T_{\mathrm{C}}$ (albeit very close if $2 k_{\mathrm{F}} \ll \xi_{0}$ ), but with a similar magnitude and the same density dependence as our analysis above. For this reason, we restricted our analysis to data taken in the range $1.1 T_{\mathrm{C}}<T<1.5 T_{\mathrm{C}}$. We remark that one obtains $\mathrm{d} \rho / \mathrm{d} T<0$ if $k_{\mathrm{F}}$ is small enough, despite the metallic conduction.

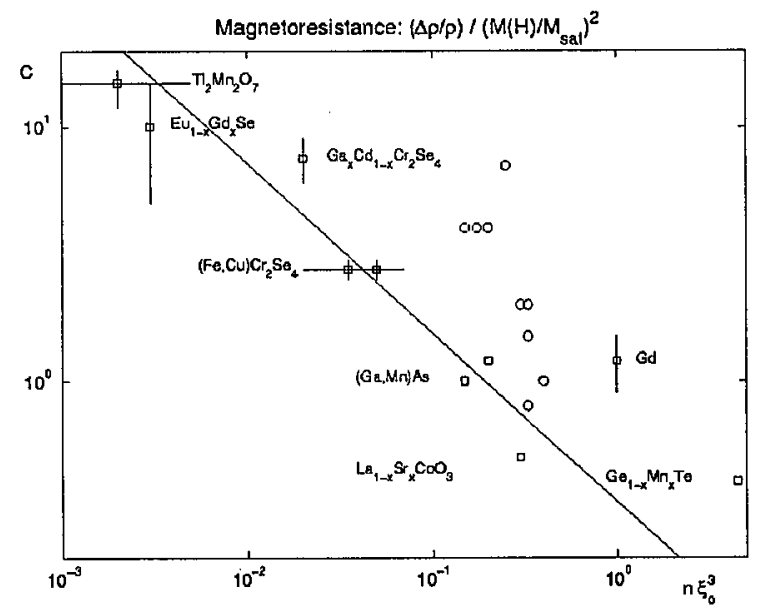

Fig. 1. Low field magnetoresistance plotted against scaled carrier density. Open circles show data from perovskite manganites, and the solid line shows a $-2 / 3$ power law. From [2], where the sources of the data are given.

*This equation refers only to that part of the resistivity arising from magnetic scattering, and for comparison to data, other processes must be subtracted out. 
Remarkably, it turns out that this scaling works rather well over different classes of materials, including the pyrochlore $\mathrm{Tl}_{2} \mathrm{Mn}_{2} \mathrm{O}_{7}$, the spinels $\mathrm{CdCr}_{2} \mathrm{Se}_{4}$, $\mathrm{Fe}_{2} \mathrm{Cr}_{2} \mathrm{~S}_{4}$, magnetic semiconductors EuSe, GeMnTe as well as simple ferromagnetic metals [2] (see Fig. 1). Of course for the latter the MR is quite small. The range of carrier density is from $10^{-3}$ to 1 .

But a conspicuous failure is for the doped perovskite manganites which have large values of $C$ together with a large carrier density. This failure is not due to the inapplicability of the weak scattering approximation. Recent numerical calculations [7] of the double exchange model have shown that even with infinite $J$, only far into the tail of the band is spin scattering strong enough to lead to localisation of the carriers. At the concentrations here $(0.1<x<0.4)$, these materials would be predicted to be good metals.

\section{Magnetic polarons}

Although the magnetoresistance (Eq. (4)) is (in our approximation) independent of the coupling constant between the conduction electrons and the magnetic fluctuations, the resistance itself is not. If the coupling constant were large enough, the Born calculation would predict a mean free path $l$ that could become arbitrarily short; the result is meaningless if it predicts $k_{\mathrm{F}} l<1$, as it must at low enough density [7]. The picture of scattering will be replaced by one of true localisation, but as we have discussed briefly above this becomes relevant even for very large $J$ only at carrier densities well below $1 \%$.

Another localising effect at low density will be a tendency to form magnetic polarons, where carrier hopping polarises a local ferromagnetic cluster of moments and thereby self-traps $[8,9]$. The problem is easiest to analyse when the fundamental origin of the magnetism separate from the conduction band carriers, as in the doped ferromagnetic semiconductors and insulators. Polarons can exist as well-defined non-overlapping entities provided $n \xi^{3} \ll 1$ (or equivalently $k_{\mathrm{F}} \xi \ll 1$ ). This regime may be reached only at temperatures somewhat above $T_{\mathrm{C}}$, even if $k_{\mathrm{F}}$ is small; at temperatures higher still, thermal fluctuations will destroy the polaron $[2,9]$. Such a localising effect will lead to saturation of the magnetoresistance, as well as a weakly non-metallic resistivity due to the easily trapped polarons.

A plausible candidate for magnetic polarons in a ferro-magnetically ordering background is the pyrochlore $\mathrm{Tl}_{2} \mathrm{Mn}_{2} \mathrm{O}_{7}$, which is a low carrier density ferromagnet $(n<1 \%)$ with a ferromagnetic coupling generated principally by superexchange [10-13]. We found elsewhere [3] that for parameters appropriate for $\mathrm{Tl}_{2} \mathrm{Mn}_{2} \mathrm{O}_{7}$, the polaron binding energy is in the range of $0.1-0.3 \mathrm{~J}$, not much larger than $T_{\mathrm{C}}$ itself. This is consistent with activated transport measured in $\mathrm{Tl}_{2-x} \mathrm{Sc}_{x} \mathrm{Mn}_{2} \mathrm{O}_{7}$ for $x>0.2$ being explained by thermal ionisation and retrapping of polarons as the mode of conduction [11].

The phenomenon described above is generic to low carrier density ferromagnets above $T_{\mathrm{C}}$, and somewhat separate from the issue of polaron or "ferron" formation at low temperatures in the ground state, which is indicative usually of phase separation between competing insulating (AF) and metallic $(F)$ phases $[14,15]$.

Ferromagnetic polarons are familiar objects in the low temperature phases of antiferromagnets, and have been studied carefully in semi-magnetic semicon- 
ductors and rare earth chalcogenides $[16,17]$. They were discussed intensively in the past in the context of the Eu chalcogenides, which have ferromagnetic or antiferromagnetic couplings depending on the chalcogen, and on the carrier concentration [16]. The special feature of the ferromagnetic polarons in a ferromagnet is, of course, that they are stable only in an intermediate temperature range and especially not in the low temperature ferromagnetic phase. Since polarons are easily trapped by disorder, this provides another mechanism for large magnetoresistance near a ferromagnetic transition.

\section{Perovskite manganites, lattice polarons and charge/orbital order}

In the perovskite manganites, it has been known for some time that the carrier density is not the sole determinant of the magnetoresistance [18] and it is now well established that dynamical electron-lattice interactions that are strong in a range of composition near $x=0.1-0.2[1,4,19-21]$.

Because the $\mathrm{Mn}^{3+}$ ion is a subject to a Jahn-Teller distortion of the cubic environment, whereas the $\mathrm{Mn}^{4+}$ ion has an empty $E_{\mathrm{g}}$ orbital, there is a competition between local charge ordering and a uniform ferromagnetic metallic phase, where the lattice distortions are suppressed. Particularly when the trivalent rare-earth ion has a small radius, a complete static charge ordering may be observed, often with a long range ordering of the nominal $\mathrm{Mn}$ (III)/ $\mathrm{Mn}$ (IV) into striped, incommensurate phases that are insulating [22]. The other extreme is observed in ordered ferromagnetic metals, when the mixed valence is suppressed. At higher temperatures, the charge- and orbitally-ordered states will "melt", while retaining the local lattice deformations and therefore some degree of charge fluctuation; it is in this phase that the bulk MR is largest. Notice that for the more metallic Sr-doped manganites $(x=0.3,0.4)$, where the lattice coupling is weak, the magnetoresistance is again small.

There are complementary viewpoints of the phenomena [21]. In any model with electron-phonon coupling the properties are determined by a dimensionless electron-phonon coupling $\lambda=g^{2} / t_{\text {eff }} \mathrm{K}$, where $g$ is the electron-phonon coupling, $t_{\text {eff }}$ the electronic bandwidth, and $K$ the relevant stiffness constant for the phonon mode. It is well known that there is a rapid crossover from the behaviour at $\lambda<1$ (band transport of carriers with a renormalised mass) to $\lambda>1$ (strong self-trapping and polaron formation). The ferromagnetic transition induced by double exchange leads to a renormalisation of the electronic bandwidth $t_{\mathrm{eff}}=t\left\langle\cos \left(\theta_{i j} / 2\right)\right\rangle$ because of the alignment of the spins; $t_{\text {eff }}$ is smaller by a numerical factor of order $0.7-0.8$ well above $T_{\mathrm{C}}$ relative to well below. Consequently there is a decrease in $\lambda$ as temperature is reduced through the transition.

In the manganites, the relevant lattice distortions are Jahn-Teller (J-T) displacements about $\mathrm{Mn}^{3+}$ ions, and large breathing distortions about the $\mathrm{Mn}^{4+}$. The pure $\mathrm{LaMnO}_{3}$ has an antiferrolastic arrangement of J-T distortions, but the frustrating effect of $\mathrm{Mn}^{4+}$ reduces the ordering transition rapidly to zero at a doping of around $20 \%$. Near and above this phase boundary, there must be strong local distortions, corresponding to a moderate $\lambda$, but there must be a range of doping where the increased bandwidth in the ferromagnetic phase reduces the coupling enough to lead to band transport. Hence on general grounds one should 
expect a metal to insulator transition accompanying the ferromagnetic order in a doping range just beyond the concentration where the long-range-ordered $\mathbf{J}-\mathbf{T}$ phase is suppressed. This broadly corresponds to what is seen in experiment.

Such a generic model ignores the cooperative nature of the lattice distortions, which are surely controlling the behaviour in detail. There is no place in this simple picture for the charge- and orbital ordering that is prominent in experiment. Further, because there is always a first-order transition between the metallic ferromagnet and the charge-ordered insulator, there is another mechanism for CMR, due to the coexistence of these two phases [23]. It turns out that coexistence is very common, and easily tuned by magnetic field [24], pressure [25], and even by light [26]. Consequently, there are regimes where the metal-insulator transition is essentially percolative in nature [23].

The two-phase coexistence is generally on a too large length scale to allow for macroscopic charge segregation, as discussed in models of ferromagnetic polarons, for example. And it is also not due to macroscopic chemical segregation; the two phases have an identical chemical composition. One possible suggestion is that the competing phases arise because of a long-range strain mismatch between the charge-ordered and metallic phases, and occurs because of "clamping" effects, familiar in ferroelectric and ferroelastic materials [27, 28].

Despite the activity of the past few years, these materials continue to yield surprises. And while the physical mechanisms are broadly understood, the details remain elusive for theoretical models.

\section{Acknowledgments}

We gratefully acknowledge discussions with G. Aeppli, B. Batlogg, C.H. Chen, S.-W. Cheong, D. Edwards, H. Hwang, A.P. Ramirez, H. Röder and Y. Tokura. Parts of the work reviewed here [2-4] were performed in collaboration with P. Majumdar, A.J. Millis and B.I. Shraiman. Work done at Cambridge University is supported by EPSRC.

\section{References}

[1] A.P. Ramirez, J. Phys., Condens. Matter 9, 8171 (1997).

[2] P. Majumdar, P.B. Littlewood, Nature 395, 479 (1998).

[3] P. Majumdar, P.B. Littlewood, Phys. Rev. Lett. 81, 1314 (1998).

[4] A.J. Millis, P.B. Littlewood, B.I. Shraiman, Phys. Rev. Lett. 74, 5144 (1995).

[5] M.E. Fisher, J.S. Langer, Phys. Rev. Lett. 20, 665 (1968).

[6] P.G. de Gennes, J. Friedel, J. Phys. Chem. Solids 4, 71 (1958).

[7] Q. Li, J. Zang, A.R. Bishop, C.M. Soukoulis, Phys. Rev. B 56, 4541 (1997).

[8] E.L. Nagaev, Physics of Magnetic Semiconductors, Mir Publishers, Moscow 1983.

[9] T. Kasuya, A. Yanase, T. Takeda, Solid State Commun. 8, 1543 (1970).

[10] Y. Shimikawa, Y. Kubo, T. Manako, Nature 379, 53 (1996).

[11] M.A. Subramanian, B.H. Toby, A.P. Ramirez, W.J. Marshall, A.W. Sleight, G.H. Kwei, Science 273, 81 (1996). 
[12] S.-W. Cheong, H.W. Hwang, B. Batlogg, L.W. Rupp, Jr., Solid State Commun. 98, 163 (1996).

[13] R. Senis, B. Martinez, J. Fontcuberta, X. Obradors, W. Cheikh-Rouhou, P. Strobel, C. Bougerol-Chaillout, M. Pernet, J. Appl. Phys. 85, 5405 (1999).

[14] M.Y. Kagan, D.I. Khomskii, M.V. Mostovoy, preprint a vailable on http://xxx.lanl.gov, cond-mat/9804213.

[15] A. Moreo, S. Yunoki, E. Dagotto, Science 283, 2034 (1999).

[16] S. Von Molnar, S. Methfessel, J. Appl. Phys. 38, 959 (1967); Y. Shapira, S. Foner, N.F. Oliveira, Jr., T.B. Reed, Phys. Rev. B 10, 4765 (1974) and references therein.

[17] See e.g. A. Golnik, J. Ginter, J.A. Gaj, J. Phys. C 16, 6073 (1983).

[18] H.Y. Hwang, S.-W. Cheong, P.G. Radaelli, M. Marezio, B. Batlogg, Phys. Rev. Lett. 75, 914 (1995).

[19] H. Röder, J. Zang, A.R. Bishop, Phys. Rev. Lett. 76, 1356 (1996).

[20] A.J. Millis, B.I. Shraiman, R. Mueller, Phys. Rev. Lett. 77, 175 (1996).

[21] A.J. Millis, Nature 392, 147 (1998).

[22] S. Mori, C.H. Chen, S.-W. Cheong, Phys. Rev. Lett 81, 3972 (1998).

[23] M. Uehara, S. Mori, C.H. Chen, S.-W. Cheong, Nature 399, 560 (1999).

[24] H. Kuwahara, Y. Tomioka, A. Asamitsu, Y. Moritomo, Y. Tokura, Science 270, 961 (1995).

[25] Y. Moritomo, H. Kuwahara, Y. Tomioka, Y. Tokura, Phys. Rev. B 55, 7549 (1997).

[26] V. Kiryukhin, D. Kasa, J.P. Hill, B. Keimer, A. Vigiliante, Y. Tomioka, Y. Tokura, Nature 386, 813 (1997); M. Fiebig, K. Miyano, Y. Tomioka, Y. Tokura, Science 280, 1925 (1998).

[27] S.R. Shenoy, T. Lookman, A. Saxena, A.R. Bishop, Los Alamos preprint, 1999.

[28] P.B. Littlewood, Nature 399, 529 (1999). 